\title{
Men's Experiences with the Hockey Fans in Training Weight Loss and Healthy Lifestyle Program
}

Stephanie Brianne Muise, ${ }^{1}$ Dawn P. Gill, ${ }^{1}$ Ashleigh De Cruz, ${ }^{1}$ Brendan Riggin, ${ }^{1}$ Roseanne Pulford, ${ }^{1}$ Shannon L. Sibbald, ${ }^{1}$ Robert J. Petrella.

\begin{abstract}
Background: Increasing rates of chronic disease, especially in men, have led to an increased effort to implement lifestyle interventions focusing on healthy eating and physical activity. Men are underrepresented in lifestyle programs and some studies have observed that males prefer men-only programs that occur in the context of sports. This paper reviews men's feedback regarding motivation for joining and overall experience in a 12-week lifestyle intervention in the context of junior level ice hockey teams. Methods: Men age 35-65 with a BMI $\geq 28$ were recruited from local ice hockey team fan bases in London and Sarnia Ontario, Canada and randomized to the 12-week lifestyle intervention or control group. Those who attended at least 6 of the 12 weekly sessions, including at least one session in the final six weeks $(n=30)$ were asked to complete an online questionnaire upon finishing the active phase of the intervention. The questionnaire elicited reasons for joining the program, changes seen following their participation, and the usefulness of specific components of the program. Results: For the 27 men who completed the questionnaire, weight loss and a desire to increase physical activity were the two main reasons cited for joining the program. After the intervention, $100 \%$ of the men reported eating a healthier diet and $78 \%$ increased their activity level. Program satisfaction was high and $96 \%$ of men believed both the classroom and exercise components were useful. Conclusion: Our results support previous research showing increased levels of satisfaction in men when lifestyle interventions are run in a sporting context and incorporate both an educational component and an exercise component.
\end{abstract}

Keywords: Life Style; Exercise; Diet; Sports (Source: MeSH-NLM).

About the Author: Stephanie Muise is a fourth and final year medical student at Western University's schulich School of Medicine in London, Ontario, Canada. She was also awarded the Maunder Scholarship from the Heart and Stroke Foundation for pursuit of research in cardiovascular medicine and a schulich Research Opportunities Program grant to help provide funding for this project.

\section{Introduction}

Rates of chronic disease have been rising in both men and women and it is now considered a worldwide burden.' Obesity is a widely recognized risk factor for many of these chronic illnesses, including cardiovascular disease, hypertension, dyslipidemia, type 2 diabetes and some cancers. The prevalence of being overweight or obese is higher in Canadian males than females ( $62 \%$ compared with $46 \%$ ), placing them at further risk (Statistics Canada. Available from: http://www.statcan.gc.ca/tables-tableaux/sum-som/lo1/cstor/health81b-eng.htm, updated 2016 March 07; cited 2016 May 10). There are numerous factors that may contribute to this disparity, including the fact that men are less knowledgeable than women about health, disease and their specific lifestyle risk factors and they also are less likely to access or apply health information. ${ }^{2,3}$ Additionally, men tend to be less aware of their overweight status and may associate increased body size with masculinity. ${ }^{4}$ Cultural constructs fuel this issue by tying men's health-related behavior, including seeking help, alcohol consumption and diet, to their degree of masculinity. ${ }^{5}$ Therefore, in order for lifestyle interventions geared towards men to be successful, they must incorporate these cultural ideals of masculinity. ${ }^{6}$

Evaluation of lifestyle interventions has provided some knowledge foundation for effective strategies to combat obesity; however, most of these studies have primarily involved women.? There remains a paucity of research investigating the motivations that men have to lose weight, as well as how best to engage them in weight loss behaviours. ${ }^{89}$ Currently, reviews of men's perceptions of weight loss programs have revealed that they often consider them inherently feminized domains, ${ }^{10}$ which decreases their appeal and serves as a barrier to participation. There is some evidence to suggest that men who want to lose weight may be more attracted to lifestyle programs that encompass competition-driven physical activity in the company of other males that they can identify with. ${ }^{8,11} \mathrm{~A}$ recent systematic review showed that lifestyle interventions that are situated in a sporting context, driving feelings of affiliation and belonging, result in higher levels of satisfaction and lower dropout rates. ${ }^{12}$

Being a sports fan creates powerful social and psychological connections that may be drawn upon when designing weight loss programs for men..$^{13} \mathrm{~A}$ research group in the United Kingdom used this premise to design the Football Fans in Training (FFIT) program, which encouraged men to lead a healthier lifestyle through a sports-related medium." The results from their pragmatic randomized controlled trial (RCT) demonstrated the acceptability of the program to high risk men, as well as the effectiveness of the program in leading to sustained weight

${ }^{1}$ Schulich School of Medicine \& Dentistry, Western University, Ontario, Canada. 
loss 12-months after the program. 14,15 In collaboration with the research team who developed FFIT, we have developed Hockey Fans in Training (Hockey FIT) - adapted from the successful FFIT program to suit Canadian hockey fans and also incorporating Healthesteps, an evidence-based, lifestyle prescription program supported by coaching and eHealth tools. ${ }^{16}$ This paper is a qualitative analysis of the experiences of the men enrolled in the Hockey FIT pilot RCT based on responses to a feedback questionnaire administered after the active phase of the intervention.

Ice hockey is core to Canadian culture: two thirds of adult Canadians follow the game as a fan and 8/10 identify ice hockey as a key part of what it means to be Canadian. ${ }^{17}$ In terms of numbers of fans, access to teams in small/large communities, infrastructure of team organizations, media exposure, and facilities to engage in exercise and education surrounding lifestyle change, junior hockey arenas and facilities provided an ideal venue to pilot the Hockey FIT program. Also important is that sport venues may provide an opportunity to engage those at higher risk for lifestyle-associated chronic disease. Specifically, one survey suggested that sports fans weigh more, eat higher fat foods and have worse general health habits than their nonsports fan counterparts (Available from: http://news.health. com/2008/12/23/sports-fans-not-as-healthy/, updated 2008 Dec 23; cited 2016 May 10). Together, these facts made it ideal to conduct our two-arm pragmatic pilot RCT (Hockey FIT) in two Junior A Hockey Teams in London and Sarnia Ontario.

In brief, Hockey FIT is a gender-sensitized lifestyle intervention program consisting of 12 weekly, 90-minute sessions, delivered by 2 coaches to 20 men (i.e. ratio 1:10) at each of the 2 sites. The sessions incorporated both an education and exercise component and took place either in the Junior A hockey team's arena or their training facility (Goodlife Fitness) in London or Sarnia. The education components involved interactive (non-didactic) discussions led by trained coaches who were graduate students in Kinesiology. Each week they followed specific guidelines from a coaching manual, which corresponded to a manual that the participants were given at the start of the intervention. These educational sessions involved discussing topics related to weight management, including healthy eating, alcohol consumption, increasing physical activity and SMART (Specific Measurable Attainable Realistic Timely) goal setting. Following this, Hockey FIT coaches lead structured hockey-related exercise sessions where men received training in aerobic and strength and flexibility exercises tailored to individual fitness levels, abilities and pre-existing health conditions. Across sessions, Hockey FIT coaches provided instruction on the behaviour change techniques shown to be effective in physical activity and dietary interventions at these sessions (i.e. self-monitoring of weight and exercise, specific goal setting, feedback on behaviour) and promoted peer support. Men also received individualized lifestyle prescriptions for exercise and healthy eating based on their baseline level of fitness and diet. ${ }^{16,18-22}$ Following the 12-week active phase, there is a 40-week "maintenance phase", where participants continue to independently build on their exercise prescriptions, set new goals and track their exercise, physical activity, and healthy eating with the support of the eHealth technology tools that were introduced in week 12 of the active phase. This remote technological support has previously been used to help participants maintain longterm behaviour changes. ${ }^{16,23}$

Hockey FIT included components designed to appeal to male hockey supporters (and which were found to be successful with FFIT), including: team-based incentives (i.e., T-shirts, jerseys, jackets, tours of the hockey players' dressing rooms and lounges along with surprise visits from team and local professional hockey players); elements of competition (i.e., quizzes); a session focusing on alcohol consumption; and coach-led encouragement of positive banter (i.e., hockey-related, often ironic or self-deprecatory jokes), thus actively facilitating the use of humour to help men address serious or sensitive topics (i.e., weight gain) that they may otherwise be reluctant to discuss with others.

While the quantitative outcomes of the pilot RCT will be reported elsewhere, the aims of this paper are to report the perceptions and experiences of men who enrolled in the Hockey FIT pilot pragmatic RCT in the context of: 1) motivations for joining the program; 2) satisfaction with the program components; and 3) suggestions for improvement of the program.

\section{Methods}

The Hockey FIT pilot RCT was registered with ClinicalTrials.gov Protocol Registration and Results System. A detailed description of the study design can be found in the recently published protocol paper. ${ }^{24}$ Participants in the Hockey FIT pilot RCT were recruited from the local Hockey Team fan base using email blasts, social media, and print media at hockey events in arenas. The inclusion criteria required the participants to be male, between ages 35-65 with a body-mass index (BMI) of at least $28 \mathrm{~kg} / \mathrm{m}^{2}$. Additionally, they must have cleared the Physical Activity Readiness Questionnaire (PAR-Q; Available from: http:// www.csep.ca/CMFiles/publications/parq/par-q.pdf) or received clearance from a health care provider. The exclusion criteria included being unable to comprehend the letter of information and consent documentation.

A total of 40 men were randomized using a computer-based random number sequence to the Hockey FIT intervention group; 20 from each of London and Sarnia, as shown in Figure 1. A program questionnaire was administered after the 12-week Hockey FIT active phase intervention to participants who had attended a minimum of six Hockey FIT coaching sessions ( $50 \%$ ), including at least one of the sessions in the final six weeks of the intervention. It was a modified version of the questionnaire that was administered in the FFIT study at their 12-month follow-up and consisted of 14 questions that were designed to elicit reasons that the men joined the program, goals that they had achieved and any feedback about what they liked and disliked about the program. They were given the option to select all responses that applied for each question. The questionnaire was designed using an online Qualtrics survey program ${ }^{25}$ so that the men would be able to complete the questionnaire online after being sent an individualized link via email. The men were given information about when they would receive the link, as well as how to complete the survey at their week 11 Hockey FIT coaching session. They were again reminded about the questionnaire at their final Hockey FIT coaching session and the emails containing the questionnaire links were 
Table 1. Baseline Characteristics of Hockey FIT Intervention Group

\begin{tabular}{lc}
\hline Characteristic & Intervention $(\mathrm{N}=40)$ \\
\hline Age, median (IQR) & $48.0(41.5-57.0)$ \\
\hline White ethnicity, $\mathrm{n}(\%)$ & $38(95.0)$ \\
\hline Marital status: Married or common-law, n (\%) & $35(87.5)$ \\
\hline Educational status, $\mathrm{n}(\%)$ & $3(7.5)$ \\
Did not finish high school & $10(25.0)$ \\
High school diploma or equivalent & $14(35.0)$ \\
Certificate/diploma (‘Bachelor's) & $13(32.5)$ \\
$\geq$ Bachelor's Degree & $112.5(102.5-127.2)$ \\
\hline Weight (kg), median (IQR) & $116.5(111.9-125.6)$ \\
\hline Waist circumference (cm), median (IQR) & $34.9(31.9-38.6)$ \\
\hline Body mass index (kg/m²), median (IQR) & $6,860(3,254)$ \\
\hline Steps/day (7 day average), mean (SD) & $8(6-9)$ \\
\hline STC summary score, median (IQR) &
\end{tabular}

$\mathrm{IQR}=$ interquartile range; $\mathrm{SD}=$ standard deviation; $\mathrm{STC}=$ Starting the Convention (Dietary Q: ranges from o to 16 , lower $=$ healthier diet)

Table 2. Reasons for Joining Hockey FIT as Civen by the Intervention Group

\begin{tabular}{lc}
\hline \multicolumn{1}{c}{ Reason for joining } & $\begin{array}{c}\text { Questionnaire responders } \\
(\mathbf{N}=27), \mathrm{n}(\%)\end{array}$ \\
\hline To lose weight & $24(88.9)$ \\
\hline To increase your physical activity & $23(85.2)$ \\
\hline To learn how to eat more healthily & $20(74.0)$ \\
\hline To increase your energy level & $14(51.9)$ \\
\hline To improve your mood & $8(29.6)$ \\
\hline
\end{tabular}

Table 3. Changes Noticed by Participants After Completion of the 12 Week Active Intervention

\begin{tabular}{lc}
\hline \multicolumn{1}{c}{$\begin{array}{c}\text { Changes seen by participants } \\
\text { at 12-weeks }\end{array}$} & $\begin{array}{c}\text { Questionnaire responders } \\
(\mathrm{N}=27), \mathrm{n}(\%)\end{array}$ \\
\hline I have lost $5-10 \%$ of my body weight & $10(37.0)$ \\
\hline I have increased my physical activity level & $27(77.8)$ \\
I have started eating a healthier diet & $27(100.0)$ \\
\hline I feel better about myself & $20(74.0)$ \\
\hline
\end{tabular}

sent out that day. A single reminder email was sent out to men who had yet to complete their questionnaire, one week after their final coaching session. Additionally, men were reminded of the questionnaire when they met with the research team for their 12-week measurement sessions.

The quantitative outcome measures from the Hockey FIT pilot RCT which are reported elsewhere, include the primary outcome of weight loss (absolute and \%) and several secondary outcomes: 1) objectively measured clinical characteristics (BMI, waist circumference, resting blood pressure); 2) self-reported physical activity (7-day average steps using a pedometer, and both a total physical activity score and sitting time from International Physical Activity Questionnaire25); 3) self-reported eating habits (total healthful eating score from the Starting the Conversation tool, ${ }^{26}$ as well as a fatty food score, sugary food
Figure 1. Distribution of Participants Across Intervention and Control Groups and Proportion of Intervention and Program Questionnaire Completion

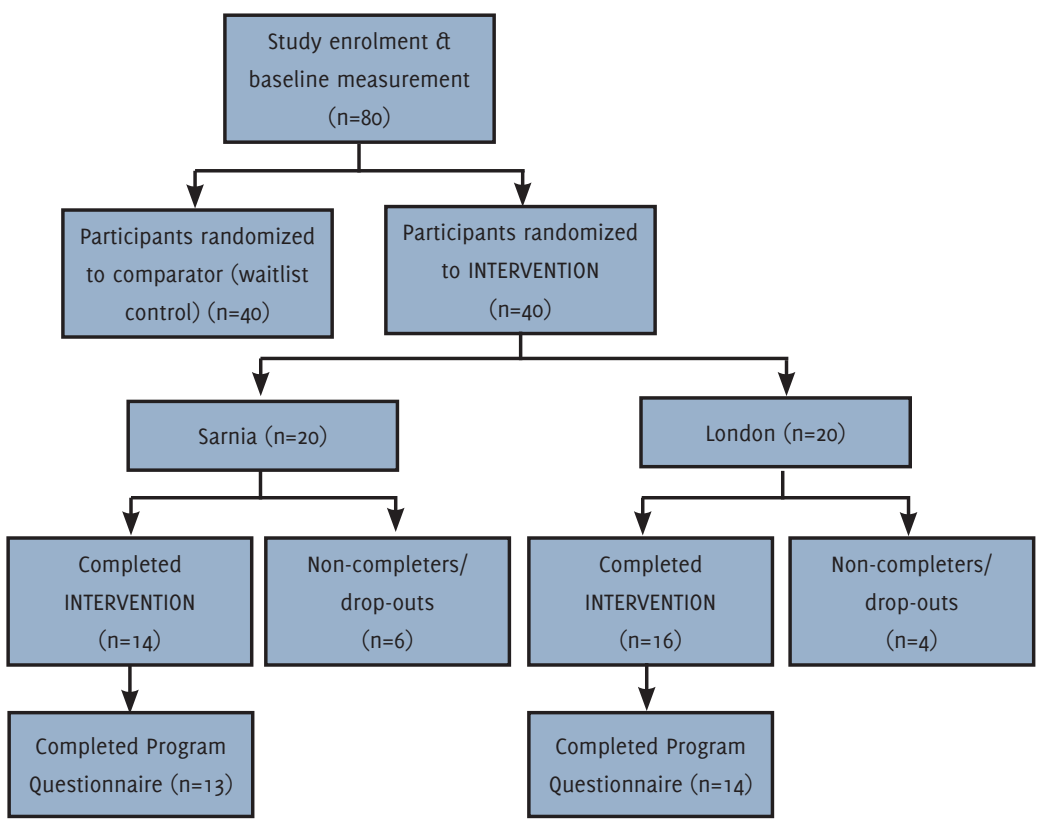

Diagram illustrating the distribution of participants across intervention and control groups, and proportion that completed both the intervention and the Program Questionnaire across two sites in London and Sarnia Ontario, Canada. Completed INTERVENTION was defined as participants who attended at least six sessions (50\%), including at least one session in the final six weeks..

score, and fruit and vegetable consumption from the Dietary Instrument for Nutrition Education questionnaires) ${ }^{27}$ and alcohol consumption (7-day recall diary).28 and 4) psychological and health-related quality of life measures (self-esteem score from the Rosenberg Self Esteem Scale, ${ }^{29-30}$ affect scores from the International Positive and Negative Affect Schedule Short Form, ${ }^{31}$ and self-rated health from the European Quality of Life - 5 Dimensions Questionnaire - 3 Level Version).32

The Western University Health Sciences Research Ethics Board approved this study on April 06, 2015 (Approval \#106310), and all participants provided written informed consent.

\section{Results}

A total of 30 men across the two implementation sites (London and Sarnia) met the attendance criteria and were sent the program questionnaire (Figure 1). The average age of the participants was 48 years and the majority were of white ethnicity and married. The average baseline weight was $112.5 \mathrm{~kg}$, BMI 34.9 and waist circumference $116.5 \mathrm{~cm}$ (Table 1). Of these men, 27 ( $90 \%$ ) completed the questionnaire and the majority ( $78 \%$ ) had completed between $9-11$ of the 12 in-person training sessions.

The most common reasons stated for participants joining the Hockey FIT program were to lose weight $(89 \%)$, increase physical activity $(85 \%)$ and eat more healthily $(74 \%)$ (Table 2$)$. In reporting what changed over the course of the 12 weeks, $100 \%$ of the men indicated that they began to eat a healthier diet, $78 \%$ said they had increased their physical activity, $74 \%$ felt better about themselves and $37 \%$ stated they had lost $5-10 \%$ of their body weight (Table 3). 
Participants were also asked about which aspects of the program were most beneficial in helping them lose weight, become more fit and feel better. Ninety six percent of the men felt that both the exercise and educational classroom components of the evening sessions were beneficial. In terms of physical activity incentives, the pedometer, the exercise prescriptions and goal setting, as well as the actual evening exercise sessions were all found to be "quite" or "very" useful by $85 \%$ of the participants. With respect to efforts aimed at improving eating habits, the two aspects of the program deemed most useful by participants were information on appropriate portion sizes and information regarding the importance of eating regular meals, including breakfast. In contrast, learning details about how to understand food labels was ranked only slightly useful by $25 \%$ of the participants. Participants also found support from others useful in their quest for a healthier lifestyle. Hearing about how other men were doing was ranked as quite or very useful by $85 \%$ of the men, as was getting family support in changing what they ate or drank and their level of physical activity.

In the free text areas where men could add in their own ideas about what they liked about the program, it was a common theme to hear about the great organization and preparedness of the coaches. They enjoyed the company of other men who had similar interests to themselves and enjoyed the fact that the exercise sessions incorporated some elements of hockey and competition. Many men stressed that they would have liked the sessions to be 2 hours instead of 1.5 in order to include a full hour of exercise, and often men suggested having more than one session each week.

\section{Discussion}

The top two motivations for joining the program were losing weight and increasing physical activity, which aligns with the previously described notion of men joining lifestyle interventions with the main two goals of improving fitness as well as physical appearance. ${ }^{9}$ However, the importance of wanting to learn how to eat more healthily cannot be overlooked and reflects previous studies examining the preference of men for programs that include both education on healthy eating, as well as an exercise component. ${ }^{33}$ Competition was introduced into some of the exercise sessions through use of team-based activities and was considered useful in helping the men increase their level of engagement during the exercises. This has been widely described in previous literature as a beneficial tool to encourage participant involvement. ${ }^{33-35}$ The element of competition also extended to the success of the pedometer in motivating men to become more active on their own as it gave them an external representation of how they were comparing to themselves and others on a daily basis.

Interestingly, although the men enjoyed the education on healthy eating that they received during the classroom sessions, the most informative aspect was visualizing appropriate portion sizes. This is in alignment with findings from the FFIT studies in Scotland and may indicate that controlling portion size is the most effective way to help men lose weight. Although some men found the nutrition label education useful, others thought that they would not be able to practically incorporate this into their lives due to the excess time and effort that reading labels requires. Time-efficient strategies are critical to success in designing weight-loss programs for men as it has been demonstrated that time and energy constraints are considered the main barriers to engaging in lifestyle interventions. ${ }^{36}$ There is the potential for bias in the results reported here because the responses recorded are only from those men who attended greater than $50 \%$ of the sessions and were motivated enough to provide feedback through an online survey. Men who met the attendance criteria but did not respond (10\%) may have not had the same opinion as those described here. Additionally, we did not collect responses from those participants who attended less than $50 \%$ of the sessions, as we did not feel as though they would be able to adequately describe their experience with the various components of Hockey FIT. These men were contacted to answer a separate telephone survey, which detailed their reasons for not completing the study and will be reported elsewhere.

Attaining support from other men and the coaches was also ranked as an important contributor to achieving success in the program. Although support is widely cited as a positive influence when attempting to lose weight, the support from colleagues in Hockey FIT was unique in that it came from others with similar BMIs and recreational interests. This may have made the men feel more comfortable with sharing their ideas and more able to give others tangible advice on overcoming barriers or setbacks. Importantly though, support from family in changing what the men ate or drank was ranked as the most important factor in achieving weight loss success. This is an important point to acknowledge because as MacLean et al. suggested, it is often the women in the household that do the food purchasing and prepare the meals. ${ }^{37}$ Since women in the household appear to have the greatest control over the type of foods that the men eat, portion control is an important element of diet that men can regulate themselves.

In addressing the men's comments about increasing the length of the exercise portion per session and the number of sessions per week, although this arrangement would likely result in an increase in weight lost, it could also increase the disparity in the number of sessions individual participants could attend. One of the draws of the Hockey FIT program is how achievable it is for men to come to a session only once per week, learn about techniques to incorporate healthy diet and exercise into their own schedules, and then independently use these tools to modify their own lifestyles. Increasing the number of sessions per week may be achievable for some, but it also discourages the sustainability of the results achieved, as there is less responsibility placed on the individuals themselves. Increasing the exercise portion of the sessions is a valid consideration, and perhaps one to consider in future iterations of the program.

In summary, this pilot RCT was designed to assess the acceptability and feasibility of a 12-week healthy lifestyle intervention in the professional hockey team setting. A program questionnaire was administered at the completion of the active phase of the intervention and captured the main reasons that men joined the program, their satisfaction with elements of the program as well as suggestions for improvement. Although there were some suggestions as to how to improve the program in the future, it was widely reported as a positive experience with many of the men wishing it could be extended for longer than 12 weeks. 


\section{References}

1. Bloom DE, Cafiero ET, Jané-Llopis E, Abrahams-Gessel S, Bloom LR, Fathima $S$, et al. The global economic burden of noncommunicable diseases. Geneva: World Economic Forum; 2011.

2. Courtenay WH. Engendering health: a social constructionist examination of men's health beliefs and behaviors. Psychology of Men at Masculinity. 2000;1(1):4-15.

3. Galdas PM, Cheater F, Marshall P. Men and health help-seeking behavior: literature review. J Adv Nurs. 2005 Mar;49(6):616-23.

4. Smith JA, Braunack-Mayer A, Wittert G. What do we know about men's help-seeking and health service use? Med J Aust. 2006 Jan;184(2):81-3.

5. de Visser R, Smith JA. Mister in-between: a case study of masculine identity and health-related behaviour. J Health Psychol. 2006 Sep;11(5):685-95.

6. Morgan PJ, Young MD, Smith JJ, Lubans DR. Targeted health behavior interventions promoting physical activity: a conceptual model. Exerc Sport Sci Rev. 2016 Apr;44(2):71-80

7. Bottorff JL, Seaton CL, Johnson ST, Caperchione CM, Oliffe JL, More K, et al. An updated review of interventions that include promotion of physical activity for adult men. Sports Med. 2015 Jun;45(6):775-800.

8. Sabinsky MS, Toft U, Raben A, Holm L. Overweight men's motivations and perceived barriers towards weight loss. Eur J Clin Nutr. 2007 Apr;61(4):526-31. 9. Hankey CR, Leslie WS, Lean ME. Why lose weight? Reasons for seeking weight loss by overweight but otherwise healthy men. Int J Obes Relat Metab Disord. 2002 Jun;26(6):880-2.

10. Gough B. 'Real men don't diet': an analysis of contemporary newspaper representations of men, food and health. Soc Sci Med. 2007 Jan;64(2):326-37. 11. Gray CM, Hunt K, Mutrie N, Anderson AS, Leishman J, Dalgarno L, et al. Football Fans in Training: the development and optimization of an intervention delivered through professional sports clubs to help men lose weight, become more active and adopt healthier eating habits. BMC Public Health. 2013 Mar;13:232.

12. Robertson C, Archibald D, Avenell A, Douglas F, Hoddinott $P$, van Teijlingen $E$, et al. Systematic reviews of and integrated report on the quantitative, qualitative and economic evidence base for the management of obesity in men. Health Technol Assess. 2014 May;18(35):v-vi, xxiii-xxix, 1-424.

13. Hirt ER, Clarkson JJ. The psychology of fandom: understanding the etiology, motives, and implications of fanship. In: Kahle LR, Close AG, editors. Consumer behavior knowledge for effective sports and event marketing. New York: Routledge/Taylor a Francis Group; 2011. p. 59-85.

14. Hunt K, Wyke S, Gray CM, Anderson AS, Brady A, Bunn C, et al. A gender-sensitised weight loss and healthy living programme for overweight and obese men delivered by Scottish Premier League football clubs (FFIT): a pragmatic randomised controlled trial. Lancet. 2014 Apr 5;383(9924):1211-21. 15. Hunt K, Gray CM, Maclean A, Smillie S, Bunn C, Wyke S. Do weight management programmes delivered at professional football clubs attract and engage high risk men? A mixed-methods study. BMC Public Health. 2014 Jan 21;14:50.

16. Gill DP, Blunt W, Bartol C, Pulford RW, De Cruz A, Simmavong PK, et al. HealtheSteps ${ }^{\mathrm{T} M}$ Study Protocol: a pragmatic randomized controlled trial promoting active living and healthy lifestyles in at-risk Canadian adults delivered in primary care and community-based clinics. BMC Public Health. 2017 Feb $7 ; 17(1): 173$.

17. The Environics Institute for Social Change. Hockey in Canada - 2012 Public Opinion Survey. The Environics Institute for Social Change; 2012.

18. Petrella RJ, Lattanzio CN, Overend TJ. Physical activity counseling and prescription among canadian primary care physicians. Arch Intern Med. 2007 Sep 10; 167(16):1774-81.

19. Petrella RJ, Aizawa K, Shoemaker K, Overend T, Piche L, Marin M, et al. Efficacy of a family practice-based lifestyle intervention program to increase physical activity and reduce clinical and physiological markers of vascular health in patients with high normal blood pressure and/or high normal blood glucose (SNAC): study protocol for a randomized controlled trial. Trials. 2011 Feb $16 ; 12: 45$.

20. Stuckey MI, Knight E, Petrella RJ. The step test and exercise prescription tool in primary care: a critical review. Crit Rev Phys Rehabil Med. 2012;24(12):109-23.

21. Michie S, Abraham C, Whittington C, McAteer J, Gupta S. Effective techniques in healthy eating and physical activity interventions: a meta-regression. Health Psychol. 2009 Nov;28(6):690-701.

22. Artinian NT, Fletcher GF, Mozaffarian D, Kris-Etherton P, Van Horn L, Lichtenstein $\mathrm{AH}$, et al. Interventions to promote physical activity and dietary lifestyle changes for cardiovascular risk factor reduction in adults: a scientific statement from the American Heart Association. Circulation. 2010 Jul 27;122(4):406-41.

23. Stuckey MI, Shapiro S, Gill DP, Petrella RJ. A lifestyle intervention supported by mobile health technologies to improve the cardiometabolic risk profile of individuals at risk for cardiovascular disease and type 2 diabetes: study rationale and protocol. BMC Public Health. 2013 Nov 7;13:1051.

24. Gill DP, Blunt W, De Cruz A, Riggin B, Hunt K, Zou G, et al. Hockey Fans in Training (Hockey FIT) pilot study protocol: a gender-sensitized weight loss and healthy lifestyle program for overweight and obese male hockey fans. BMC Public Health. 2016 Oct 19;16(1):1096.

25. Craig CL, Marshall AL, Sjöström M, Bauman AE, Booth ML, Ainsworth BE, et al. International physical activity questionnaire: 12-country reliability and validity. Med Sci Sports Exerc. 2003 Aug;35(8):1381-95.

26. Paxton AE, Strycker LA, Toobert DJ, Ammerman AS, Glasgow RE. Starting the conversation performance of a brief dietary assessment and intervention tool for health professionals. Am J Prev Med. 2011 Jan;40(1):67-71.

27. Roe L, Strong C, Whitesdie C, Neil A, Mant D. Dietary intervention in primary care: validity of the DINE method for diet assessment. Fam Pract. 1994 Dec;11(4):375-81.

28. Emslie C, Lewars H, Batty CD, Hunt K. Are there gender differences in levels of heavy, binge and problem drinking? Evidence from three generations in the west of Scotland. Public Health. 2009 Jan;123(1):12-4.

29. Rosenberg M. Society and adolescent self-image. Princeton, NJ: Princeton University Press; 1965.

30. Gray-Little B, Williams V, Hancock T. An item response theory analysis of the Rosenberg Self-Esteem Scale. Pers Soc Psychol Bull. 1997;23(5):443-51.

31. Thompson ER. Development and validation of an internationally reliable short-form of the Positive and Negative Affect Schedule (PANAS). J Cross Cult Psychol. 2007 Mar;38(2):227-42.

32. EuroQol Group. EuroQol--a new facility for the measurement of health-related quality of life. Health Policy. 1990 Dec;16(3):199-208.

33. Hooker SP, Wilcox S, Rheaume CE, Burroughs EL, Friedman DB. Factors related to physical activity and recommended intervention strategies as told by midlife and older African American men. Ethn Dis. 2011 Summer;21(3):261-7. 34. Griffith DM, Allen J0, Johnson-Lawrence V, Langford A. Men on the move: a pilot program to increase physical activity among African American men. Health Educ Behav. 2014 Apr;41(2):164-72.

35. Robertson S, Zwolinsky S, Pringle A, McKenna J, Daly-Smith A, White A. 'It is fun, fitness and football really': a process evaluation of a football-based health intervention for men. Qualitative Research in Sport, Exercise and Health. $2013 \operatorname{Sep}$ 3;5(3):419-39.

36. Gavarkovs AG, Burke SM, Petrella RJ. The physical activity-related barriers and facilitators perceived by men living in rural communities. Am J Mens Health. 2015 Jul 31. [Epub ahead of print]

37. MacLean A, Hunt K, Gray C, Smillie S, Wyke S. How do men's female relatives feature in their accounts of changing eating practices during a weight-management programme delivered through professional football clubs? Int J Mens Health. 2014;13(2):121-38.

\section{Acknowledgments}

Football Fans in Training (FFIT) team: Prof Kate Hunt, PhD; Prof Sally Wyke, PhD; Cindy M Gray, PhD; Christopher Bunn, PhD.

\section{Conflict of Interest Statement at Funding}

Summer Research Opportunities Program via Schulich School of Medicine; Movember Foundation Men's Health and Wellbeing Challenge Grant.

\section{Author Contributions}

Conceptualization, Approval of the final version: SBM, DPG, ADC, BR, RP, RJP. Data collection: SBM, ADC, BR, RP. Data analysis and interpretation, Critical revision of the manuscript: SBM, DPG, RJP. Writing, Contribution of patients or study materials, Funding acquisition: SBM. Administrative/technical advice: DPG.

Cite as:

Muise SB, Gill DP, De Cruz A, Riggin B, Pulford R, Sibbald SL, Petrella RJ. Men's experiences with the Hockey Fans in Training weight loss and healthy lifestyle program. Int J Med Students. 2016 Sep-Dec;4(3):91-5. 